\title{
Three New Diterpenoid Alkaloids from Roots of Aconitum ouvrardianum HAND.-MazZ.
}

\author{
Lang-Huan Hou, Dong-Lin Chen, Xi-Xian JiAn, and Feng-Peng WANG* \\ Department of Chemistry of Medicinal Natural Products and Key Laboratory of Drug Targeting of Education Ministry \\ PRC, West China College of Pharmacy, Sichuan University; No. 17, Duan 3, Renmin Nan Road, Chengdu 610041, P. R. \\ China. Received February 9, 2007; accepted March 17, 2007
}

\begin{abstract}
$A$ new $\mathrm{C}_{19}$-diterpenoid alkaloid, ouvrardiantine (1) and two new $\mathrm{C}_{20}$-diterpenoid alkaloids, ouvrardiandines A (2) and B (3) were isolated from the root of Aconitum ouvrardianum HAND.-MAzz. The structure of the new alkaloids was established on the basis of spectral data (1D- and 2D-NMR, HR-MS).
\end{abstract}

Key words Aconitum ouvrardianum; Ranunuclaceae; diterpenoid alkaloid; ouvrardiantine; ouvrardiandine A; ouvrardiandine B

The plant Aconitum ouvrardianum HAND.-MAzz. grows in Diqing country of Yunnan province in China at an elevation of $3000 \mathrm{~m}$. Biological activity studies performed on diterpenoid alkaloids of "CaoWu" species have shown analgesia, anti-imflammatory and antiarrhythmic action. ${ }^{1,2}$ To our knowledge, no phytochemical study of this plant has previously been undertaken. These reasons and our interest in the species prompted us to work with the A. ouvrardianum. In the course of our studies on the alkaloids of this plant, a new $\mathrm{C}_{19}$-diterpenoid alkaloid, ouvrardiantine (1) and two new $\mathrm{C}_{20}$ diterpenoid alkaloids, ouvrardiandines A (2) and B (3) have been isolated from the root of $A$. ouvrardianum. This paper describes the separation and structural elucidation of these new alkaloids.

\section{Results and Discussion}

Ouvrardiantine (1) was obtained as a white amorphous powder, $[\alpha]_{\mathrm{D}}^{20}+25^{\circ}\left(c=0.8, \mathrm{CHCl}_{3}\right)$, whose molecular formula was determined as $\mathrm{C}_{35} \mathrm{H}_{49} \mathrm{NO}_{11}$ by HR-ESI-MS $(\mathrm{m} / \mathrm{z}$ $\left.660.3388[\mathrm{M}+\mathrm{H}]^{+}\right)$. The NMR spectral of 1 exhibited characteristic features of an aconitine-type $\mathrm{C}_{19}$-diterpenoid alkaloid $^{3)}$ bearing an $N$-ethyl group $\left(\delta_{\mathrm{H}} 1.16,3 \mathrm{H}, \mathrm{t}, J=7.2 \mathrm{~Hz} ; \delta_{\mathrm{C}}\right.$ $12.2 \mathrm{q}, 48.8 \mathrm{t})$, four methoxyl groups $\left(\delta_{\mathrm{H}} 3.18,3.27,3.31\right.$, 3.52 , each $3 \mathrm{H}$, s; $\delta_{\mathrm{C}} 58.1 \mathrm{q}, 59.0 \mathrm{q}, 56.0 \mathrm{q}, 58.8 \mathrm{q}$ ), an acetyl group $\left(\delta_{\mathrm{H}} 1.36,3 \mathrm{H}, \mathrm{s} ; \delta_{\mathrm{C}} 21.6 \mathrm{q}, 169.8 \mathrm{~s}\right)$, and an anisoyl group $\left(\delta_{\mathrm{H}} 6.91,8.01\right.$, each $2 \mathrm{H}$, $\mathrm{AA}^{\prime} \mathrm{BB}^{\prime}$ system, $J=8.8 \mathrm{~Hz}$; $\left.\delta_{\mathrm{H}} 3.87,3 \mathrm{H}, \mathrm{s} ; \mathrm{Ar}-\mathrm{OCH}_{3}\right)$. The four methoxyl groups were located at $\mathrm{C}-1, \mathrm{C}-6, \mathrm{C}-16$, and $\mathrm{C}-18$, respectively, on the basis of the $\mathrm{HMBC}$ correlations between $1-\mathrm{OCH}_{3}\left(\delta_{\mathrm{H}} 3.31\right)$
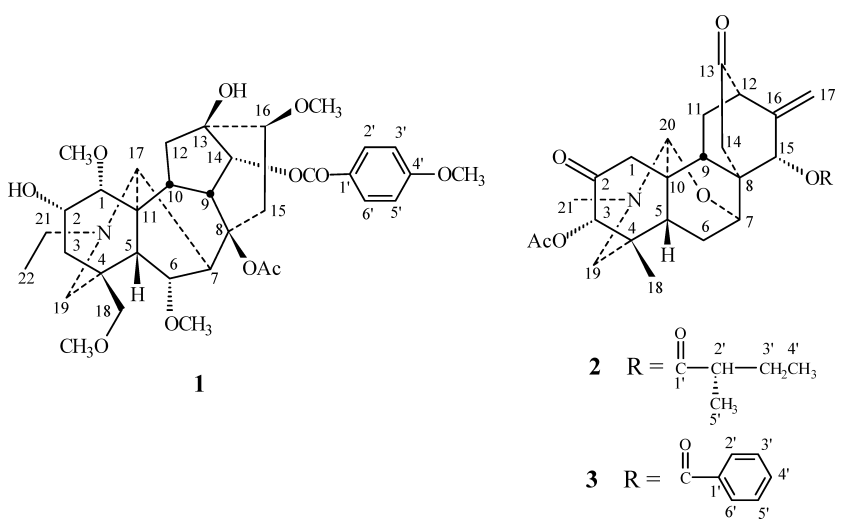

Fig. 1. The Structure of Compounds $\mathbf{1}-\mathbf{3}$ and $\mathrm{C}-1\left(\delta_{\mathrm{C}} 85.6 \mathrm{~d}\right), 6-\mathrm{OCH}_{3}\left(\delta_{\mathrm{H}} 3.18\right)$ and C-6 $\left(\delta_{\mathrm{C}} 82.2 \mathrm{~d}\right)$, $16-\mathrm{OCH}_{3}\left(\delta_{\mathrm{H}} 3.52\right)$ and $\mathrm{C}-16\left(\delta_{\mathrm{C}} 83.7 \mathrm{~d}\right)$, and $18-\mathrm{OCH}_{3}\left(\delta_{\mathrm{H}}\right.$ $3.27)$ and $\mathrm{C}-18\left(\delta_{\mathrm{C}} 79.1 \mathrm{t}\right)$. The one-proton doublet signal at $\delta_{\mathrm{H}} 4.88(1 \mathrm{H}, \mathrm{d}, J=5.2 \mathrm{~Hz})$ was assigned to $\mathrm{H}-14 \beta,{ }^{3)}$ as well as the upfield proton signal of the acetyl at $\delta_{\mathrm{H}} 1.36$ due to the shielding effect of the $14 \alpha$-OAs, suggesting the presence of 14-anisoyl group and the acetyl group at $\mathrm{C}-8 .{ }^{3)}$ In the ${ }^{1} \mathrm{H}$ NMR, the downfield chemical shift of $16-\mathrm{OCH}_{3}\left(\delta_{\mathrm{H}} 3.52\right.$, $3 \mathrm{H}, \mathrm{s})$ in addition to the doublet signal of $\mathrm{H}-14$ indicated the presence of hydroxyl group at C-13. ${ }^{3)}$ Meanwhile, another hydroxyl group was located at $\mathrm{C}-2$, based on the chemical shifts $\left(\delta_{\mathrm{C}} 62.3 \mathrm{~d}\right)$ of this carbon and the HMBC correlations of H-2 $\left(\delta_{\mathrm{H}} 4.01\right)$ with C-4 $\left(\delta_{\mathrm{C}} 38.9 \mathrm{~s}\right)$ and C-11 $\left(\delta_{\mathrm{C}} 52.7 \mathrm{~s}\right)$. The configuration of 2-OH was $\alpha$-orientation, based on the downfield shift of C-19 $\left(\delta_{\mathrm{C}} 51.8 \mathrm{t}\right)$ in the ${ }^{13} \mathrm{C}-\mathrm{NMR}$ spectra of compound 1. In this case, there was a hydrogen bond formed between $\mathrm{N}$ atom and $2 \alpha-\mathrm{OH}$ group (Fig. 2). ${ }^{4}$ Finally, all the evidence given above led to assignment of the structure of ouvrardiantine as 1 .

Ouvrardiandine A (2) was isolated as a white amorphous powder, $[\alpha]_{\mathrm{D}}^{20}-62^{\circ}\left(c=0.3, \mathrm{CHCl}_{3}\right)$. The HR-ESI-MS of 2 exhibited a protonated molecular ion peak at $\mathrm{m} / z$ 500.2647 $[\mathrm{M}+\mathrm{H}]^{+}$(Calcd 500.2643) corresponding to the pseudo-molecular formula of $\mathrm{C}_{28} \mathrm{H}_{37} \mathrm{NO}_{7}$. The ${ }^{1} \mathrm{H}$ - and ${ }^{13} \mathrm{C}$-NMR spectra of 2 exhibited characteristic features of an atisine-type alkaloid. ${ }^{5)}$ Its NMR data showed the presence of an $N$-methyl group $\left(\delta_{\mathrm{H}} 2.36,3 \mathrm{H}, \mathrm{s} ; \delta_{\mathrm{C}} 41.8 \mathrm{q}\right)$, two ketonic carbons $\left(\delta_{\mathrm{C}}\right.$ $202.6 \mathrm{~s}, 209.5 \mathrm{~s})$, an exo-methylene $\left(\delta_{\mathrm{H}} 5.10,5.24\right.$, each $1 \mathrm{H}$, $\left.\mathrm{s} ; \delta_{\mathrm{H}} 115.9 \mathrm{t}\right)$, a methyl group $\left(\delta_{\mathrm{H}} 1.29,3 \mathrm{H}, \mathrm{s} ; \delta_{\mathrm{C}} 22.3 \mathrm{q}\right)$, an acetyl $\left(\delta_{\mathrm{H}} 2.14,3 \mathrm{H}, \mathrm{s} ; \delta_{\mathrm{C}} 170.7 \mathrm{~s}, 21.1 \mathrm{q}\right)$, and a 2-methylbutyryl ester group $\left(\delta_{\mathrm{H}} 0.92,3 \mathrm{H}, \mathrm{t}, J=7.3 \mathrm{~Hz} ; 1.16,3 \mathrm{H}, \mathrm{d}, J=\right.$ $7.1 \mathrm{~Hz} ; 1.48,1.72,2 \mathrm{H}, \mathrm{m} ; 2.41,1 \mathrm{H}, \mathrm{m} ; \delta_{\mathrm{C}} 11.7 \mathrm{q}, 16.7 \mathrm{q}$, $26.5 \mathrm{t}, 41.4 \mathrm{~d}, 176.5 \mathrm{~s})$. Its ${ }^{1} \mathrm{H}$ - and ${ }^{13} \mathrm{C}-\mathrm{NMR}$ spectra also showed the distinctive $\mathrm{N}, \mathrm{O}$-mixed acetal moiety $\left(\delta_{\mathrm{H}} 4.33\right.$, $\left.1 \mathrm{H}, \mathrm{s} ; \delta_{\mathrm{C}} 93.4 \mathrm{~d}\right)$. The two ketonic ${ }^{13} \mathrm{C}$-signals at $\delta_{\mathrm{C}} 209.5 \mathrm{~s}$ and $\delta_{\mathrm{C}} 202.6 \mathrm{~s}$ were attributable to $\mathrm{C}-13$ and $\mathrm{C}-2$, respec-

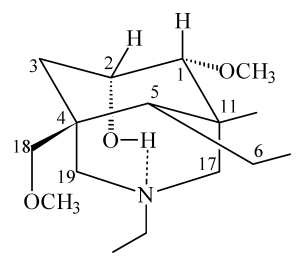

Fig. 2. The Hydrogen Bond Formation between $\mathrm{N}$ Atom and $2 \alpha-\mathrm{OH}$ of $\mathbf{1}$ 
Table 1. ${ }^{1} \mathrm{H}$ - and ${ }^{13} \mathrm{C}-\mathrm{NMR}$ Spectral Data of Compound $\mathbf{1}$

\begin{tabular}{|c|c|c|c|c|c|}
\hline Carbon & $\delta_{\mathrm{C}}$ & $\delta_{\mathrm{H}}$ mult. $(J=\mathrm{Hz})$ & Carbon & $\delta_{\mathrm{C}}$ & $\delta_{\mathrm{H}}$ mult. $(J=\mathrm{Hz})$ \\
\hline 1 & $85.6 \mathrm{~d}$ & $3.12 \mathrm{~d}(4.8)$ & 17 & $60.7 \mathrm{~d}$ & $2.80 \mathrm{brs}$ \\
\hline 2 & $62.3 \mathrm{~d}$ & $4.01 \mathrm{~m}$ & 18 & $79.1 \mathrm{t}$ & $3.01 \mathrm{~d}(8.4)(\alpha)$ \\
\hline \multirow[t]{2}{*}{3} & $42.1 \mathrm{t}$ & $1.74 \mathrm{dd}(14.8,3.6)(\alpha)$ & & & $3.65 \mathrm{~d}(8.4)(\beta)$ \\
\hline & & $1.96 \mathrm{dd}(15.0,2.2)(\beta)$ & 19 & $51.8 \mathrm{t}$ & $2.53,2.58 \mathrm{ABq}(11.4)$ \\
\hline 4 & $38.9 \mathrm{~s}$ & & 21 & $48.8 \mathrm{t}$ & $2.61,2.68 \mathrm{~m}$ \\
\hline 5 & $49.4 \mathrm{~d}$ & $2.23 \mathrm{~d}(6.4)$ & 22 & $12.2 \mathrm{q}$ & $1.16 \mathrm{t}(7.2)$ \\
\hline 6 & $82.2 \mathrm{~d}$ & $4.01 \mathrm{~d}(6.0)$ & $1-\mathrm{OCH}_{3}$ & $56.0 \mathrm{q}$ & $3.31 \mathrm{~s}$ \\
\hline 7 & $49.7 \mathrm{~d}$ & $3.10 \mathrm{br} \mathrm{s}$ & $6-\mathrm{OCH}_{3}$ & $58.1 \mathrm{q}$ & $3.18 \mathrm{~s}$ \\
\hline 8 & $85.3 \mathrm{~s}$ & & $16-\mathrm{OCH}_{3}$ & $58.8 \mathrm{q}$ & $3.52 \mathrm{~s}$ \\
\hline 9 & $45.6 \mathrm{~d}$ & $2.97 \mathrm{~m}$ & $18-\mathrm{OCH}_{3}$ & $59.0 \mathrm{q}$ & $3.27 \mathrm{~s}$ \\
\hline 10 & $40.8 \mathrm{~d}$ & $2.20 \mathrm{~m}$ & $8-\mathrm{OAc}$ & $169.8 \mathrm{~s}$ & \\
\hline 11 & $52.7 \mathrm{~s}$ & & & $21.6 \mathrm{q}$ & $1.36 \mathrm{~s}$ \\
\hline \multirow[t]{2}{*}{12} & $37.7 \mathrm{t}$ & $2.18 \mathrm{~m}(\alpha)$ & 14-OAs & & \\
\hline & & $2.68 \mathrm{~m}(\beta)$ & $\mathrm{CO}$ & $166.1 \mathrm{~s}$ & \\
\hline 13 & $74.7 \mathrm{~s}$ & & $1^{\prime}$ & $122.6 \mathrm{~s}$ & \\
\hline 14 & $78.4 \mathrm{~d}$ & $4.88 \mathrm{~d}(5.2)$ & $2^{\prime}, 6^{\prime}$ & $131.7 \mathrm{~d}$ & $8.01 \mathrm{~d}(8.8)$ \\
\hline \multirow[t]{2}{*}{15} & $39.5 \mathrm{t}$ & $2.46 \mathrm{dd}(14.2,5.6)(\alpha)$ & $3^{\prime}, 5^{\prime}$ & $113.8 \mathrm{~d}$ & $6.91 \mathrm{~d}(8.8)$ \\
\hline & & $2.97 \mathrm{~m}(\beta)$ & $4^{\prime}$ & $163.5 \mathrm{~s}$ & \\
\hline 16 & $83.7 \mathrm{~d}$ & $3.30 \mathrm{~m}$ & $4^{\prime}-\mathrm{OCH}_{3}$ & $55.4 \mathrm{q}$ & $3.87 \mathrm{~s}$ \\
\hline
\end{tabular}

tively, based on $\mathrm{HMBC}$ correlations of C-13 with $\mathrm{H}-14\left(\delta_{\mathrm{H}}\right.$ $2.40,2.92)$ and $\mathrm{C}-2$ with $\mathrm{H}-1\left(\delta_{\mathrm{H}} 2.28\right)$, respectively. The acetoxyl group and the 2-methylbutyryl ester group could be assigned at $\mathrm{C}-3$ and $\mathrm{C}-15$, respectively, as a result of the HMQC and the HMBC correlations of $\mathrm{H}-3\left(\delta_{\mathrm{H}} 4.54\right)$ with $\mathrm{COCH}_{3}\left(\delta_{\mathrm{C}} 170.7, \mathrm{~s}\right), \mathrm{C}-4\left(\delta_{\mathrm{C}} 39.4, \mathrm{~s}\right), \mathrm{C}-5\left(\delta_{\mathrm{C}} 56.8, \mathrm{~d}\right)$, and H-15 $\left(\delta_{\mathrm{H}} 5.56\right)$ with CO-methylbutyryl $\left(\delta_{\mathrm{C}} 176.5, \mathrm{~s}\right), \mathrm{C}-16$ $\left(\delta_{\mathrm{C}} 142.4, \mathrm{~s}\right), \mathrm{C}-17\left(\delta_{\mathrm{C}} 115.9, \mathrm{t}\right)$, respectively. In the NOE spectrum of 2 , the irradiation of H-3 $\left(\delta_{\mathrm{H}} 4.54\right)$ led to the enhancement of the signals of H-5 $\left(\delta_{\mathrm{H}} 2.02\right)$ and H-18 $\left(\delta_{\mathrm{H}}\right.$ $1.29)$, indicating that the 3 -acetoxyl group was $\alpha$-oriented. Meanwhile, the positive correlation of $\mathrm{H}-15\left(\delta_{\mathrm{H}} 5.56\right)$ with H-9 $\left(\delta_{\mathrm{H}} 2.41\right)$ also confirmed the $\alpha$-configuration of the 15 (2-methylbutyryl) ester group. In addition, the stereochemistry of this group may be assigned as " $S$ " based on references, which reported some other natural products bearing the same moiety, ${ }^{6-9)}$ and the absolute configuration of $\mathrm{C}-2^{\prime}$ was in agreement with the L-isoleucine pathway in the biosynthesis of the secondary metabolites. ${ }^{10)}$ The structure of ouvrardiandine A thus was deduced as $\mathbf{2}$ by careful analysis of the 1D-NMR and 2D-NMR $\left({ }^{1} \mathrm{H}-{ }^{1} \mathrm{H}\right.$ COSY, HMQC, $\mathrm{HMBC}$ and NOE) spectra.

Ouvrardiandine B (3) was a white amorphous powder, $[\alpha]_{\mathrm{D}}^{20}-17.6^{\circ}\left(c=0.2, \mathrm{CHCl}_{3}\right)$. The pseudo-molecular formula $\mathrm{C}_{30} \mathrm{H}_{33} \mathrm{NO}_{7}$ was inferred from its HR-ESI-MS $(\mathrm{m} / \mathrm{z}$ $542.2133[\mathrm{M}+\mathrm{Na}]^{+}$, Calcd 542.2149). The ${ }^{1} \mathrm{H}-$ and ${ }^{13} \mathrm{C}-$ NMR spectra of $\mathbf{3}$ were very similar to that of $\mathbf{2}$ except for the lack of a 2-methylbutyryl ester group, but gave the distinctive signals of a benzoyl group $\left(\delta_{\mathrm{H}} 7.47,7.62,8.03 ; \delta_{\mathrm{C}}\right.$ see Table 2). Comparison of the ${ }^{13} \mathrm{C}$-NMR and MS data of 2 and $\mathbf{3}$ determined the structure of ouvrardiandine $\mathrm{B}$ to be $\mathbf{3}$.

\section{Experimental}

General Silica gel $\mathrm{H}$ and $\mathrm{GF}_{254}$ (Qingdao Haiyang Chemical Group Co., China) were used for column chromatography and TLC, respectively. The spots were detected by Dragendorff reagent. Optical rotation measurements were made using a Perkin-Elmer 341 polarimeter. ${ }^{1} \mathrm{H}$ - and ${ }^{13} \mathrm{C}-\mathrm{NMR}$ spectra were recorded on a Varian Unity INOVA 400/45 and Bruker Avance 600 spectrometer in $\mathrm{CDCl}_{3}$ with TMS as internal standard. HR-ESI-MS was measured on a VG Auto Spec 3000 mass spectrometer. IR was measured on a Nicolet FI-IR 200SXY spectrophotometer.

Plant Material Aconitum ouvrardianum Hand.-Mazz. was collected in
Table 2. ${ }^{1} \mathrm{H}$ - and ${ }^{13} \mathrm{C}$-NMR Spectral Data of Compounds $\mathbf{2}$ and $\mathbf{3}$

\begin{tabular}{|c|c|c|c|c|}
\hline \multirow{2}{*}{ Carbon } & \multicolumn{2}{|r|}{2} & \multicolumn{2}{|r|}{3} \\
\hline & $\delta_{\mathrm{C}}$ & $\delta_{\mathrm{H}}$ mult. $(J=\mathrm{Hz})$ & $\delta_{\mathrm{C}}$ & $\delta_{\mathrm{H}}$ mult. $(J=\mathrm{Hz})$ \\
\hline \multirow[t]{2}{*}{1} & $47.7 \mathrm{t}$ & 2.28 brs $(\alpha)$ & $47.7 \mathrm{t}$ & 2.37 brs $(\alpha)$ \\
\hline & & $2.29 \operatorname{brs}(\beta)$ & & 2.37 brs $(\beta)$ \\
\hline 2 & $202.6 \mathrm{~s}$ & & $202.5 \mathrm{~s}$ & \\
\hline 3 & $79.7 \mathrm{~d}$ & $4.54 \mathrm{~s}$ & $79.8 \mathrm{~d}$ & $4.57 \mathrm{~s}$ \\
\hline 4 & $39.4 \mathrm{~s}$ & & $39.5 \mathrm{~s}$ & \\
\hline 5 & $56.8 \mathrm{~d}$ & $2.02 \mathrm{brs}$ & $56.8 \mathrm{~d}$ & $2.07 \mathrm{brs}$ \\
\hline \multirow[t]{2}{*}{6} & $43.2 \mathrm{t}$ & $1.62 \mathrm{~d}(12.0)(\alpha)$ & $43.2 \mathrm{t}$ & $1.67 \mathrm{~d}(12.0)(\alpha)$ \\
\hline & & $\begin{array}{l}2.05 \mathrm{dd} \\
\quad(12.0,6.0)(\beta)\end{array}$ & & $\begin{array}{l}2.10 \mathrm{dd} \\
\quad(12.0,6.0)(\beta)\end{array}$ \\
\hline 7 & $74.1 \mathrm{~d}$ & $4.03 \mathrm{~d}(6.0)$ & $74.1 \mathrm{~d}$ & $4.05 \mathrm{dd}(6.0)$ \\
\hline 8 & $52.9 \mathrm{~s}$ & & $53.0 \mathrm{~s}$ & \\
\hline 9 & $39.0 \mathrm{~d}$ & $2.41 \mathrm{~m}$ & $39.0 \mathrm{~d}$ & $2.50 \mathrm{~m}$ \\
\hline 10 & $42.4 \mathrm{~s}$ & & $42.9 \mathrm{~s}$ & \\
\hline \multirow[t]{2}{*}{11} & $26.4 \mathrm{t}$ & $1.83 \mathrm{~m}(\alpha)$ & $26.4 \mathrm{t}$ & $1.88 \mathrm{~m}(\alpha)$ \\
\hline & & $2.20 \mathrm{~m}(\beta)$ & & $2.27 \mathrm{~m}(\beta)$ \\
\hline 12 & $53.8 \mathrm{~d}$ & $3.10 \mathrm{t}(2.9)$ & $53.8 \mathrm{~d}$ & $3.19 \mathrm{t}(2.8)$ \\
\hline 13 & $209.5 \mathrm{~s}$ & & $209.6 \mathrm{~s}$ & \\
\hline \multirow[t]{2}{*}{14} & $39.4 \mathrm{t}$ & $2.40 \mathrm{~d}$ (hidden) $(\alpha)$ & $39.6 \mathrm{t}$ & $2.59 \mathrm{~d}(19.6)(\alpha)$ \\
\hline & & $2.92 \mathrm{~d}(19.0)(\beta)$ & & $3.04 \mathrm{~d}(19.6)(\beta)$ \\
\hline 15 & $75.0 \mathrm{~d}$ & $5.56 \mathrm{~s}$ & $76.0 \mathrm{~d}$ & $5.85 \mathrm{~s}$ \\
\hline 16 & $142.4 \mathrm{~s}$ & & $141.9 \mathrm{~s}$ & \\
\hline \multirow[t]{2}{*}{17} & $115.9 \mathrm{t}$ & $5.10 \mathrm{~s}$ & $116.5 \mathrm{t}$ & $5.24 \mathrm{~s}$ \\
\hline & & $5.24 \mathrm{~s}$ & & $5.32 \mathrm{~s}$ \\
\hline 18 & $22.3 \mathrm{q}$ & $1.29 \mathrm{~s}$ & $22.3 \mathrm{q}$ & $1.31 \mathrm{~s}$ \\
\hline 19 & $49.7 \mathrm{t}$ & $2.77,2.84 \mathrm{ABq}(10.2)$ & $49.7 \mathrm{t}$ & $2.79,2.85 \mathrm{ABq}(10.2)$ \\
\hline 20 & $93.4 \mathrm{~d}$ & $4.33 \mathrm{~s}$ & $93.4 \mathrm{~d}$ & $4.35 \mathrm{~s}$ \\
\hline 21 & $41.8 \mathrm{q}$ & $2.36 \mathrm{~s}$ & $41.9 \mathrm{q}$ & $2.39 \mathrm{~s}$ \\
\hline \multirow[t]{2}{*}{ 3-OAc } & $170.7 \mathrm{~s}$ & & $170.8 \mathrm{~s}$ & \\
\hline & $21.1 \mathrm{q}$ & $2.14 \mathrm{~s}$ & $21.2 \mathrm{q}$ & $2.14 \mathrm{~s}$ \\
\hline \multicolumn{5}{|l|}{ 15-Ester } \\
\hline $\mathrm{CO}$ & $176.5 \mathrm{~s}$ & & & \\
\hline $2^{\prime}$ & $41.4 \mathrm{~d}$ & $2.41 \mathrm{~m}$ & & \\
\hline $3^{\prime}$ & $26.5 \mathrm{t}$ & $1.48,1.71 \mathrm{~m}$ & & \\
\hline $4^{\prime}$ & $11.7 \mathrm{q}$ & $0.92 \mathrm{t}(7.3)$ & & \\
\hline $5^{\prime}$ & $16.7 \mathrm{q}$ & $1.16 \mathrm{~d}(7.1)$ & & \\
\hline \multicolumn{5}{|l|}{$15-\mathrm{OBz}$} \\
\hline $\mathrm{CO}$ & & & $166.4 \mathrm{~s}$ & \\
\hline $1^{\prime}$ & & & $129.2 \mathrm{~s}$ & \\
\hline $2^{\prime}, 6^{\prime}$ & & & $129.8 \mathrm{~d}$ & $8.03 \mathrm{~d}(8.0)$ \\
\hline $3^{\prime}, 5^{\prime}$ & & & $128.6 \mathrm{~d}$ & $7.47 \mathrm{t}(8.0)$ \\
\hline $4^{\prime}$ & & & $133.6 \mathrm{~d}$ & $7.62 \mathrm{t}(8.0)$ \\
\hline
\end{tabular}


the Diqing country of Yunnan province, China. The plant was identified taxonomically by Professor Qin-Er Yang (Institute of Botany, Chinese Academy of Sciences, Beijing). A voucher specimen was deposited in the herbarium of the West China College of Pharmacy, Sichuan University.

Extraction and Isolation of Alkaloids Powdered roots (1.6 kg) of Aconitum ouvrardianum were percolated with $0.2 \% \mathrm{HCl}$ until 401 was collected. The filtrate was then alkalized with $\mathrm{NH}_{4} \mathrm{OH}$ to $\mathrm{pH}>9$, extracted with ethyl acetate three times, and evaporated to give the total crude alkaloids $(18.0 \mathrm{~g})$. The crude alkaloids $(8.0 \mathrm{~g})$ were chromatographed on silica gel columns using gradient elution with petroleum ether-acetone $(10: 1 \rightarrow 4: 1)$ to give fractions I-IV. Fraction II $(300 \mathrm{mg})$ was separated on a silica gel column with cyclohexane-acetone $(20: 1)$ to give compound 1 ( $8 \mathrm{mg})$. Silica gel column chromagraphy of Fraction III $(500 \mathrm{mg})$ was eluted with ether-acetone (10:1) to give $\mathbf{2}(5 \mathrm{mg})$ and $\mathbf{3}(4.5 \mathrm{mg})$.

Ouvrardiantine (1): White amorphous powder, $[\alpha]_{\mathrm{D}}^{20}+25^{\circ}\left(c=0.8, \mathrm{CHCl}_{3}\right)$, IR $(\mathrm{KBr}) \mathrm{cm}^{-1}$ : 3491, 1718, 1607, 1512, 1459, 1258, 1099; ${ }^{1} \mathrm{H}-\mathrm{NMR}(600$ $\mathrm{MHz})$ and ${ }^{13} \mathrm{C}-\mathrm{NMR}(150 \mathrm{MHz})$ : see Table 1; HR-ESI-MS: $m / z 660.3388$ $[\mathrm{M}+\mathrm{H}]^{+}$, Calcd for $\mathrm{C}_{35} \mathrm{H}_{50} \mathrm{NO}_{11} 660.3378$.

Ouvrardiandine A (2): White amorphous powder, $[\alpha]_{\mathrm{D}}^{20}-62^{\circ}(c=0.3$, $\left.\mathrm{CHCl}_{3}\right)$, IR (KBr) cm ${ }^{-1}: 3445,1731,1462,1364,1234,1045 ;{ }^{1} \mathrm{H}-\mathrm{NMR}$ $(600 \mathrm{MHz})$ and ${ }^{13} \mathrm{C}-\mathrm{NMR}(150 \mathrm{MHz})$ : see Table 2; HR-ESI-MS: $m / z$ 500.2647 $[\mathrm{M}+\mathrm{H}]^{+}$, Calcd for $\mathrm{C}_{28} \mathrm{H}_{38} \mathrm{NO}_{7} 500.2643$

Ouvrardiandine $\mathrm{B}(3)$ : White amorphous powder, $[\alpha]_{\mathrm{D}}^{20}-17.6^{\circ}(c=0.2$, $\mathrm{CHCl}_{3}$ ), IR (KBr) cm ${ }^{-1}: 3445,1718,1602,1515,1454,1269,1108,1045$;
${ }^{1} \mathrm{H}-\mathrm{NMR}(400 \mathrm{MHz})$ and ${ }^{13} \mathrm{C}-\mathrm{NMR}(100 \mathrm{MHz})$ : see Table 2; HR-ESI-MS: $m / z 542.2133[\mathrm{M}+\mathrm{Na}]^{+}$, Calcd for $\mathrm{C}_{30} \mathrm{H}_{33} \mathrm{NNaO}_{7} 542.2149$.

\section{References}

1) Lin L. Y., Chen Q. H., Wang F. P., West China J. Pharm. Sci., 19, 200-205 (2004).

2) Ameri A., Prog. Neurobiol., 56, 211-235 (1998).

3) Pelletier S. W., Mody N. V., Joshi B. S., Schramm L. C., "Alkaloids: Chemical and Biological Perspectives," Vol. 2, ed. by Pelletier S. W., Wiley, New York, 1984, pp. 205-642.

4) Ding L. S., Chen W. X., Nat. Prod. R\&D, 1, 6-32 (1989).

5) Wang F. P., Liang X. T., "Alkaloids: Chemistry and Biology," Vol. 59, ed. by Cordell G. A., Elsevier, U.S.A., 2002, pp. 1-104.

6) Wada K., Bardo H., Kawahara N., Heterocycles, 31, 1081-1088 (1990).

7) Pelletier S. W., Dailey O. D., Jr., Moy N. V., J. Org. Chem., 46, 3284 3293 (1981).

8) Shen X. L., Zhou X. L., Chen Q. H., Chen D. L., Wang F. P., Chem. Pharm. Bull., 50, 1265-1267 (2002).

9) Stefan O., Helena R., Carbohydr. Res., 284, 271-277 (1996).

10) Alvarez-Garcia R., Torres-Valencia J. M., Roman L. U., Hernandez J. D., Cerda-Garcia-Rojas C. M., Joseph-Nathan P., Phytochemistry, 66, $639-642$ (2005). 